\title{
Conducta alimentaria de poblaciones de Triatoma dimidiata (Hemiptera: Reduviidae: Triatominae) en una zona endémica y sus implicaciones epidemiológicas
}

Triatoma dimidiata populations' (Hemiptera: Reduviidae:

Triatominae) feeding behaviour in an endemic zone and related epidemiological implications

Ana E. Farfán-García y Víctor M. Angulo-Silva

Centro de Investigaciones en Enfermedades Tropicales. CINTROP. Universidad Industrial de Santander. elvirafarfan01@hotmail.com, pitorio@hotmail.com

Recibido 5 Noviembre 2009/Enviado para Modificación 25 Enero 2011/Aceptado 2 Febrero 2011

\section{RESUMEN}

Objetivo Determinar la conducta alimentaria de Triatoma dimidiata en hábitats domiciliarios y extradomiciliarios en una zona endémica de Santander, Colombia. Materiales y métodos Mediante la técnica de ELISA, se procesaron 367 contenidos intestinales de insectos capturados en zonas rurales de los municipios de Capitanejo y Macaravita. Estos fueron procesados por medio de la técnica de ELISA, con 12 antisueros policlonales anti-especie animal específicos. Los hospederos de $T$. dimidiata fueron determinados por la reactividad ante cada antisuero; se establecieron las proporciones de hospederos.

Resultados El 42,2 \% de los contenidos intestinales procesados fueron reactivos en el ELISA para proteínas sanguíneas de uno o más de 10 hospederos. En la totalidad de los insectos reactivos de intradomicilio y peridomicilio se identificaron proteínas de animales domésticos, con mayor frecuencia la sangre de gallina, seguida de caprino, canino y humano; en los insectos silvestres también se detectó sangre de animales domésticos como cabra, gallina y equino. En los insectos intradomésticos, sangre de animales como fara y armadillo. El hospedero humano fue identificado en domicilio y peridomicilio en el $11 \%$ de los insectos. Conclusiones Se determinó el carácter ecléctico del vector en hábitats domiciliarios y extradomiciliarios, por la identificación de sangre humana, de animales silvestres y domésticos. Se evidenció movilidad de las poblaciones silvestres hacia el domicilio y peridomicilio por el hallazgo de sangre de animales domésticos en éstos y de animales silvestres en los insectos domésticos y peridomésticos. Estos resultados contribuyen a la comprensión de los ciclos de transmisión de $T$. cruzi.

Palabras Clave: Triatoma, conducta alimentaria, ELISA (fuente: DeCS, BIREME). 


\section{ABSTRACT}

Objective Determining Triatoma dimidiata's feeding behaviour in domiciliary and extra-domiciliary habitats in an endemic area of the Santander department in Colombia.

Materials and methods The ELISA technique was used for processing the intestinal contents of 367 insects captured in rural areas around the municipalities of Capitanejo and Macaravita. 12 anti-animal species specific polyclonal anti-sera were used in ELISA. T. dimidiata hosts were determined by reactivity to each antiserum; host percentages were established.

Results $42.2 \%$ of the intestinal content processed by ELISA was reactive for blood proteins from one or more than 10 hosts. Domestic animal proteins were identified in all reactive intra-domiciliary and peridomestic insects, most often chicken blood, followed by that from goats, canines and humans. Blood from domestic animals like goats, chicken and horses was also detected in wild insects. Blood from animals such as armadillo and fara were identified in intra-domiciliary insects. Human host protein was found in $11 \%$ of intra-domiciliary and peri-domiciliary insects.

Conclusions The vector's eclectic nature in domiciliary and extra-domiciliary habitats was determined by identifying human blood in wild and domestic animals. The wild populations' mobility towards domiciliary and peri-domiciliary areas was demonstrated by finding domestic animals' blood in them and wild animals' blood in domestic and peri-domestic insects. These results contribute towards understanding Trypanosoma cruzi transmission-cycles.

Key Words: Triatoma, feeding behaviour, ELISA (source: MeSH, NLM).

$T$ Viatoma dimidiata, presenta amplia distribución en los países de América Central y algunas zonas de Colombia, Venezuela, Ecuador y Perú; ocupa diversos hábitats domésticos, peridomésticos y silvestres tanto en áreas rurales, como en viviendas periurbanas y urbanas de poblaciones pequeñas y grandes ciudades (1-6).

En Colombia, ha sido considerado el segundo vector de mayor importancia en la transmisión de T. cruzi por su amplia distribución y frecuente hallazgo en el intradomicilio. Los pocos estudios realizados, se han limitado a indagar sobre su distribución, ensayar estrategias de control en el campo, determinar la susceptibilidad frente a insecticidas en el laboratorio y observar su conducta en condiciones de laboratorio (7-11). Poco se conoce sobre los aspectos bioecológicos y de conducta en los hábitats naturales y artificiales domésticos, que permitan determinar el riesgo para las poblaciones humanas que conviven en áreas endémicas y que conduzcan a la comprensión de los factores que condicionan el fracaso de las intervenciones de control (12). 
Aproximaciones al conocimiento de la conducta de las poblaciones de triatominos domiciliarias y extradomiciliarias, antes y después de las intervenciones de control, pueden realizarse mediante diferentes técnicas para la identificación de proteínas sanguíneas de hospederos en los contenidos intestinales de los insectos. Los estudios realizados en Centroamérica han demostrado que la aplicación de esta metodología en poblaciones de T. dimidiata, permiten conocer las asociaciones existentes con sus posibles hospederos domésticos y silvestres, el grado de movilidad entre hábitats y la relación con la transmisión de $T$. cruzi al hombre (1,13-17).

Con el objetivo de evaluar la conducta alimentaria de las poblaciones de este insecto y las posibles implicaciones epidemiológicas en hábitats domésticos y extra-domésticos en Santander, Colombia; se identificaron proteínas sanguíneas de sus hospederos en los contenidos intestinales utilizando la técnica de ELISA, previamente estandarizada en el laboratorio $(18,19)$.

\section{MATERIALES Y METODOS}

Área de estudio

Correspondió a zonas rurales de 13 veredas de los municipios de Capitanejo $\left(6^{\circ} 3 " \mathrm{~N}\right.$ y $\left.72^{\circ} 42^{\prime \prime} \mathrm{W}\right)$ y Macaravita $\left(6^{\circ} 30^{\prime \prime} \mathrm{N}\right.$ y $\left.72^{\circ} 35^{\prime \prime} \mathrm{W}\right)$ de Santander, Colombia; ubicados en las estribaciones de la cordillera oriental sobre el cañón del río Chicamocha, con alturas desde 1090 m.s.n.m hasta 2500 m.s.n.m. El paisaje se caracteriza por montañas y terrenos quebrados escarpados semiáridos, con vegetación xerofítica y múltiples cuevas formadas por la base de las montañas rocosas y acúmulos de rocas y piedras dispersas alrededor de las viviendas; potenciales refugios para vertebrados y triatominos.

Captura de triatominos

Se realizó mediante la búsqueda activa utilizando la técnica hora/hombre en el domicilio y trampas con cebo animal en el hábitat silvestre, durante los años 2002 a 2006 (20). Dentro de las viviendas se capturaron en: dormitorios, cocina, corredores, paredes externas; en el peri-domicilio: en gallineros, caneyes, corrales de cabras, hornos de barro, conejeras, camas de perros y sitios de descanso de ganado vacuno y en el área silvestre circundante: en cuevas formadas en la base de las montañas y arrumes de piedra. Los especímenes fueron identificados con la clave de Lent \& Wygodzinsky (21), disectados y su contenido intestinal almacenado en $200 \mu \mathrm{l}$ de PBS (Phosphate Buffer Saline) $0.1 \mathrm{M} \mathrm{pH} 7.2 \mathrm{a}-20^{\circ} \mathrm{C}$ hasta su procesamiento. 
Procesamiento por ELISA

Los contenidos intestinales de los insectos fueron procesados por un ELISA directo con un panel de 12 antisueros policlonales anti-especie animal de humano, bovino, equino, caprino, canino, gallina, gato, porcino, ovino, fara, armadillo y rata; según protocolo previamente estandarizado (18-19). Cada placa fue procesada con sus respectivos blancos, controles positivos y negativos; se consideraron reacciones positivas para el antisuero respectivo, valores por encima del punto de corte preestablecido; se determinaron las proporciones de hospederos identificados.

Análisis de los datos

Los datos obtenidos se registraron en una base datos en Excel (22) y los análisis se realizaron con el software Stata (23). Se determinaron los intervalos de confianza del $95 \%$ (binomial exacta) para las proporciones de hospederos identificados. Se estableció la asociación de hospederos entre los hábitats, mediante la prueba de Chi-cuadrada $\left(x^{2}\right)$, aplicando la corrección de continuidad de Yates, cuando la tabla de contingencia era de 2 x 2 y la prueba de Fisher si alguna de las frecuencias esperadas era menor de 5.

Aspectos éticos

Para el manejo de animales se siguieron las normas éticas según la Ley 84 de 1989 y la Resolución No. 008430 de 1993 del Ministerio de Protección Social de Colombia.

\section{RESULTADOS}

De los contenidos intestinales procesados, 42,2 \% (155/367) mostraron reactividad en el ELISA para proteínas sanguíneas de uno o más de los siguientes hospederos: hombre, bovino, caprino, canino, equino, gallina, gato, Didelphis marsupialis (opossum), Dasypus spp (armadillo) y Rattus rattus (rata). En el 57,8 \% de los contenidos intestinales no se identificaron fuentes sanguíneas con los antisueros disponibles, la mayoría de éstos aparentemente se encontraban en ayuno (contenido intestinal transparente y/o amarillo).

De manera general, en los 367 insectos examinados la sangre de animales domésticos fue la más encontrada (40,6\%); hospederos silvestres y humanos se detectaron en baja proporción $(4,6 \%$ y $4,7 \%)$. En cada uno de los tres hábitats los animales domésticos también constituyeron la más frecuente fuente de alimentación (peridomicilio, 62,4\%; intradomicilio, 37,8\%; silvestre, $10,9 \%)$, siendo significante la diferencia entre éstos $\left(x^{2}=148,2 \mathrm{p}=0,00\right)$. Sangre 
humana se detectó en el 4,4\% de los insectos del intradomicilio y en muy baja proporción $(0,3 \%)$ en los del peridomicilio. La sangre humana como única fuente alimentaria se observó en 3,3\% de las poblaciones de insectos, mostrando valores bajos comparados con las alimentaciones en animales domésticos $\left(x^{2}=130,9 \mathrm{p}=0,00\right)$ (Tabla 1$)$.

Tabla 1. Hospederos domésticos y silvestres identificados en poblaciones de T. dimidiata de tres hábitats en la zona de estudio

\begin{tabular}{|c|c|c|c|c|c|c|c|c|c|c|c|c|c|}
\hline \multirow{2}{*}{$\begin{array}{c}\text { Hábitat } \\
\text { de } \\
\text { captura }\end{array}$} & \multirow[t]{2}{*}{ No. } & \multicolumn{2}{|c|}{ Silv. } & \multicolumn{2}{|c|}{ Dom. } & \multicolumn{2}{|c|}{ Hum. } & \multicolumn{2}{|c|}{$\begin{array}{l}\text { Solo } \\
\text { Silv. }\end{array}$} & \multicolumn{2}{|c|}{$\begin{array}{l}\text { Solo } \\
\text { Dom. }\end{array}$} & \multicolumn{2}{|c|}{$\begin{array}{l}\text { Solo } \\
\text { Hum. }\end{array}$} \\
\hline & & $\mathrm{n}$ & $\%$ & $\mathrm{n}$ & $\%$ & $\mathrm{n}$ & $\%$ & $\mathrm{n}$ & $\%$ & $\mathrm{n}$ & $\%$ & $\mathrm{n}$ & $\%$ \\
\hline ID & 135 & 4 & 3 & 51 & 37,8 & 16 & 4,4 & 0 & 0 & 47 & 34,8 & 11 & 8,1 \\
\hline $\mathrm{P}$ & 141 & 4 & 2,8 & 88 & 62,4 & 1 & 0,3 & 0 & 0 & 84 & 59,6 & 1 & 0,7 \\
\hline$S$ & 91 & 9 & 9,9 & 10 & 10,9 & 0 & 0 & 6 & 6,6 & 7 & 7,7 & 0 & 0 \\
\hline Total & 367 & 17 & 4,6 & 149 & 40,6 & 17 & 4,7 & 6 & 1,6 & 138 & 37,6 & 12 & 3,3 \\
\hline
\end{tabular}

n:número; \%:porcentaje

$\mathrm{Al}$ analizar los insectos reactivos, capturados en el intradomicilio, se determinó ingesta de sangre de animales domésticos en el $100 \%$ de éstos, con preferencia por gallina $(43,1 \%)$; seguida de humano $31,4 \%$, canino $17,6 \%$ y caprino $15,7 \%$. En el peridomicilio, al igual que para los insectos capturados en intradomicilio, en todos los insectos reactivos se identificaron animales domésticos, siendo los más frecuentes cabra (45,5\%), gallina (42\%), canino

Tabla 2. Distribución de frecuencias de hospederos de T. dimidiata identificados entre los insectos reactivos $(n=155)$ en el ELISAa

\begin{tabular}{|c|c|c|c|c|c|c|c|c|c|c|c|}
\hline \multicolumn{4}{|c|}{ Intradomicilio } & \multicolumn{4}{|c|}{ Peridomicilio } & \multicolumn{4}{|c|}{ Silvestre } \\
\hline \multicolumn{4}{|c|}{ Insectos reactivos = 51} & \multicolumn{4}{|c|}{ Insectos reactivos $=88$} & \multicolumn{4}{|c|}{ Insectos reactivos $=16$} \\
\hline Hosp. & $\mathrm{n}$ & $\%$ & IC95 \% ${ }^{b}$ & Hosp. & $\mathrm{N}$ & $\%$ & IC95 \% ${ }^{b}$ & Hosp. & $\mathrm{n}$ & $\%$ & IC95 $\%{ }^{b}$ \\
\hline Gallina & 22 & 43,1 & $29,34-57,7$ & Cabra & 40 & 45,5 & $34,8-56,42$ & Armadillo & 7 & 43,8 & $19,8-70,1$ \\
\hline Humano & 16 & 31,4 & $19,1-45,9$ & Gallina & 37 & 42 & $31,6-53,04$ & Cabra & 6 & 37,5 & $15,2-64,6$ \\
\hline Canino & 9 & 17,6 & $8,4-30,8$ & Canino & 19 & 21,6 & $13,5-31,6$ & Gallina & 4 & 25 & $7,2-52,4$ \\
\hline $\begin{array}{l}\text { Cabra } \\
\text { Bovino }\end{array}$ & $\begin{array}{l}8 \\
6\end{array}$ & $\begin{array}{l}15,7 \\
11,8\end{array}$ & $\begin{array}{l}7.0-28.6 \\
4,4-23,9\end{array}$ & $\begin{array}{l}\text { Gato } \\
\text { Armadillo }\end{array}$ & $\begin{array}{l}6 \\
4\end{array}$ & $\begin{array}{l}6.8 \\
4,5\end{array}$ & $\begin{array}{r}2.5-14.3 \\
1,25-11,2\end{array}$ & $\begin{array}{l}\text { Equino } \\
\text { Fara }\end{array}$ & $\begin{array}{l}3 \\
3\end{array}$ & $\begin{array}{l}18.8 \\
18,8\end{array}$ & $\begin{array}{l}4.0-45,64 \\
4,0-45,64\end{array}$ \\
\hline Gato & 5 & 9,8 & $3,26-21,4$ & Bovino & 3 & 3,4 & $0,70-9,6$ & Bovino & 2 & 12,5 & $1,55-38,3$ \\
\hline $\begin{array}{l}\text { Equino } \\
\text { Armadillo }\end{array}$ & $\begin{array}{l}5 \\
2\end{array}$ & $\begin{array}{l}9.8 \\
3,9\end{array}$ & $\begin{array}{l}3.26-21.4 \\
0,47-13,5\end{array}$ & $\begin{array}{l}\text { Equino } \\
\text { Humano }\end{array}$ & $\begin{array}{l}2 \\
1\end{array}$ & $\begin{array}{l}2.3 \\
1,1\end{array}$ & $\begin{array}{l}0.27-7.96 \\
0,028-6,2\end{array}$ & $\begin{array}{l}\text { Canino } \\
\text { Gato }\end{array}$ & $\begin{array}{l}1 \\
1\end{array}$ & $\begin{array}{l}6.3 \\
6,3\end{array}$ & $\begin{array}{l}0.16-30.2 \\
0,16-30,2\end{array}$ \\
\hline Fara & 2 & 3.9 & $0.47-13.5$ & Fara & 1 & 1.1 & $0.028-6.2$ & Rata & 0 & 0 & $0-20.6$ \\
\hline Rata & 1 & 1,9 & $0,04-10,4$ & Rata & 1 & 1,1 & $0,028-6,2$ & Humano & 0 & 0 & $0-20,6$ \\
\hline Ovino & 0 & 0 & $0-6.98$ & Ovino & 0 & 0 & $0-4.1$ & Ovino & 0 & 0 & $0-20.6$ \\
\hline Porcino & 0 & 0 & $0-6,98$ & Porcino & 0 & 0 & $0-4,1$ & Porcino & 0 & 0 & $0-20,6$ \\
\hline
\end{tabular}

${ }^{a}$ Las categorías no son exhaustivamente, ni mutuamente excluyentes debido a las alimentaciones múltiples en algunos insectos, por lo tanto el porcentaje de positividad total es mayor de 100; b Intervalo de confianza del 95\% (binomial exacta); Hosp: Hospederos; n: número; \%: porcentaje 
$(21,6 \%)$ y gato $(6,8 \%)$. En el hábitat silvestre armadillo fue la fuente más común $(43,8 \%)$ además de cabra $(35,8 \%)$, gallina $(25 \%)$ y equino $(18,8 \%)$. Animales silvestres como armadillo y fara fueron detectados en los contenidos intestinales de los insectos capturados en todos los hábitats (Tabla 2).

Cuando se confrontó la proporción observada de cada hospedero entre los tres hábitats, se observaron diferencias significantes $\left(x^{2}=82,04 \mathrm{p}<0,000 \mathrm{gl}=29\right)$ en la preferencia por una o dos fuentes alimentarias; humano en intradomicilio, cabra en peridomicilio y armadillo y fara en el hábitat silvestre (Tabla 3).

Tabla 3. Distribuciones Chi-cuadrada $\left(x^{2}\right)$ en los insectos reactivos en el ELISA para los diferentes hospederos en los tres hábitats de estudio

\begin{tabular}{|c|c|c|c|c|c|c|c|}
\hline \multirow{2}{*}{ Hospedero } & ID & $\mathrm{P}$ & $S$ & \multirow{2}{*}{ Total } & \multirow{2}{*}{$\chi^{2}$} & \multirow{2}{*}{$P$} & \multirow{2}{*}{$\mathrm{gl}^{\mathrm{a}}$} \\
\hline & $\mathrm{n}$ & $\mathrm{N}$ & $\mathrm{n}$ & & & & \\
\hline Armadillo & 2 & 4 & 7 & 13 & 20,82 & 0,00 & 2 \\
\hline Fara & 2 & 1 & 3 & 6 & 9,07 & 0,01 & 2 \\
\hline Rata & 1 & 1 & 0 & 2 & 0,33 & 0,84 & 2 \\
\hline Bovino & 6 & 3 & 2 & 11 & 2,85 & 0,24 & 2 \\
\hline Cabra & 8 & 40 & 6 & 54 & 11,09 & 0,003 & 2 \\
\hline Canino & 9 & 19 & 1 & 29 & 2,96 & 0,22 & 2 \\
\hline Equino & 5 & 2 & 3 & 10 & 5,11 & 0,07 & 2 \\
\hline Gallina & 22 & 37 & 4 & 63 & 2,31 & 0,31 & 2 \\
\hline Gato & 5 & 6 & 1 & 12 & 0,32 & 0,84 & 2 \\
\hline Humano & 16 & 1 & 0 & 17 & 26,08 & 0,00 & 2 \\
\hline Total identificaciones ${ }^{b}$ & 76 & 114 & 27 & 217 & & & \\
\hline
\end{tabular}

ID: Intradomicilio; P: peridomicilio; S:silvestre; n: número; ${ }^{a}$ gl: Grados de libertad; ${ }^{b}$ Las categorías no son exhaustivamente, ni mutuamente excluyentes debido a las alimentaciones múltiples en algunos insectos, por lo tanto el número total de alimentaciones es mayor de 155. Análisis global: $\left(x^{2}=82,04 \mathrm{P}<0,000 \mathrm{gl}=29\right)$

En el $24,5 \%$ de los insectos reactivos de los diferentes hábitats fueron determinadas alimentaciones mixtas; la combinación de dos fuentes alimentarias correspondió a 16,1 \% y con tres o más se observaron en menos del $9 \%$. La identificación de dos fuentes sanguíneas fue más alta para el hábitat silvestre $(37,5 \%)$. Combinación de sangre humana con la de animales domésticos fue observada en cinco casos y la de sangre de animales silvestres con domésticos se estableció en 6,5\%. En domicilio y peridomicilio cabra, gallina y canino fueron las alimentaciones mixtas más comunes y en el hábitat silvestre, gallina con armadillo o fara y cabra con bovino, gato y equino.

\section{DISCUSION}

Los resultados de este estudio muestran la identificación de una amplia variedad de fuentes alimentarias por medio de la técnica de ELISA en los contenidos intestinales de $T$. dimidiata, que ratifica su carácter ecléctico (1,13-15). Probablemente, por el estado de ayuno de algunos insectos o por alimentaciones 
en otros hospederos de los cuales no se disponía el antisuero respectivo, 57,8 $\%$ de los insectos no mostraron reactividad ante los antisueros utilizados.

En los tres hábitats de estudio la fuente de alimentación más frecuente, significativa y preferencial fue en los animales domésticos. Aunque las proteínas sanguíneas de gallina en intradomicilio mostraron mayor proporción que las de origen humano, éstas fueron significativamente diferentes en comparación con los demás hábitats e indica que los animales domésticos y la población humana pueden ser la principal fuente de alimentación para la supervivencia de los vectores intra y extra-domiciliarios en la zona de estudio, esto coincide con los resultados de los trabajos de Panamá y Costa Rica que reportan al hospedero humano como el más prevalente en los insectos del domicilio $(1,13)$. Es importante destacar el hallazgo de sangre de animales silvestres como opossum y armadillo en $7,8 \%$ de los insectos capturados en las viviendas.

El hallazgo de $T$. dimidiata en el peridomicilio confirma la tendencia de este insecto a invadir ecótopos artificiales hechos por el hombre; los animales domésticos como perros y gallinas en sus sitios de descanso y que permanecen en este hábitat podrían actuar como una barrera en el peridomicilio ya que son las fuentes alimentarias más cercanas $(2,15)$. La alta disponibilidad de aves y mamíferos en la zona fue confirmada a partir de la información obtenida de encuestas entomológicas previas, que mostraron gran porcentaje de viviendas con gallineros y lugares de reposo para cabras y otros mamíferos en el peridomicilio, lo cual concuerda con la alta frecuencia de alimentaciones detectadas de cabra, gallina y canino (10). En este mismo hábitat se encontró sangre de armadillo, opossum y rata, similar a lo observado en el valle central de Costa Rica, en donde se identificaron ratas, ratones y marsupiales (16). En peridomicilios de una zona urbana en este mismo país, solamente seis especies de animales domésticos y silvestres de los 14 analizados en la prueba de precipitinas, fueron establecidos como hospederos de este vector (15).

En las poblaciones silvestres, se detectaron proteínas sanguíneas de animales domésticos como cabras y gallinas y de silvestres como armadillo y opossum. Similar a lo mostrado en otro trabajo, en el cual se determinaron en insectos de refugios silvestres alimentaciones en Didelphis marsupialis, aves y reptiles, el cual muestra el estrecho contacto de poblaciones de T. dimidiata silvestres con reservorios de T. cruzi, pero no con animales domésticos como en Capitanejo y Macaravita (1). 
La movilidad de la especie entre los hábitats de estudio fue evidenciada por el hallazgo de sangre humana y de animales silvestres en los insectos capturados en domicilio y peridomicilio y de sangre de animales domésticos en los del hábitat silvestre. La colonización de ecotopos artificiales y las altas tasas de infección por T. cruzi, $23 \%$ para Macaravita y $32 \%$ para Capitanejo (datos no publicados), podría aumentar el riesgo de transmisión de $T$. cruzi a humanos por el estrecho enlace del ciclo silvestre y doméstico involucrando el mismo vector; ésto coincide con los hallazgos de los estudios genéticos en T. dimidiata en la misma zona biogeográfica (12). La presencia de proteínas sanguíneas de humano y de animales domésticos en las ninfas del domicilio, así como la identificación de vectores adultos en intradomicilio con sangre de armadillo y fara, sugiere la entrada de éstos desde el exterior, probablemente originarios de ambientes silvestres, específicamente de cuevas y acúmulos de rocas localizados alrededor de las viviendas.

Diversos factores asociados con la capacidad de $T$. dimidiata para acercarse a sus hospederos, están relacionados con el tiempo que tarda en alimentarse y la capacidad alimentaria; las interrupciones frecuentes por la irritabilidad de los animales en el momento de la picadura y la disponibilidad de hospederos, hacen que el insecto se movilice en busca de otras fuentes sanguíneas para completar su alimentación. Esta conducta podría explicar las frecuentes alimentaciones múltiples (uno de cada cuatro insectos) observadas en la zona de estudio y como ha sido descrito anteriormente $(1,13,15)$.

Aunque algunos de los contenidos intestinales de T. dimidiata analizados en este estudio presentaron alto grado de digestión, la prueba de ELISA logró identificar las proteínas sanguíneas. La cantidad de proteínas identificables en el intestino por las técnicas inmunoenzimáticas dependen en gran medida del grado de digestión de las proteínas, la vitalidad, así como el tiempo transcurrido desde la alimentación del insecto hasta su disección en el laboratorio, por lo tanto, es importante tener en cuenta estos factores para el análisis de los resultados obtenidos en este tipo de estudios y para determinar de igual manera la sensibilidad y especificidad de las mismas (24).

Este trabajo contribuyó al conocimiento de la conducta de $T$. dimidiata, de las asociaciones con sus hospederos y de los mecanismos de intercambio o movilidad entre los hábitat naturales y artificiales del entorno humano. La amplia diversidad y disponibilidad de hospederos es más estable en el ambiente doméstico, principalmente en el peridomicilio, donde se hacen colonias muy 
abundantes no percibidas por sus habitantes; los cuales son excelentes refugios con gran oferta alimentaria para los insectos procedentes de hábitats silvestres. Esta característica debe tenerse en cuenta en la formulación de alternativas de control•

Agradecimientos: A las Bacteriólogas Yenny Castellanos y Laura Rodríguez, por su colaboración en la preparación de algunos antisueros, al personal del laboratorio de entomología del CINTROP y a las comunidades de las zonas muestreadas. Financiación: COLCIENCIAS. Proyecto código No. 11020413029.

\section{REFERENCIAS}

1. Zeledón R, Guardia V, Zúñiga A, Swartzwelder JC. Biology and ethiology of Triatoma dimidiata (Latreille, 1811). III. Habitat and blood sources. J Med Entomol 1973; 10:363-370.

2. Zeledón R, Montenegro VM, Zeledón O. Evidence of colonization of man-made ecotopes by Triatoma dimidiata (Latreille, 1811) in Costa Rica. Mem Inst Oswaldo Cruz 2001; 96(5):659-660.

3. Schofield CJ. Challenges of Chagas Disease vector control in Central America. Global collaboration for development of pesticides for public health, WHO/CDS/WHOPES/ GCDPP/2001.1, 14-15, p. 10-12.

4. Monroy MC, Bustamante DM, Rodas AG, Enriquez ME, Rosales RG. Habitats, dispersion and invasion of sylvatic Triatoma dimidiata (Hemiptera: Reduviidae: Triatominae) in Peten, Guatemala. J Med Entomol 2003; 40:800-806.

5. Dorn P, Monroy C, Curtis A. Triatoma dimidiata (Latreille, 1811): A review of its diversity across its geographic range and the relationship among populations. Infection, Genetics and Evolution 2007; 7:343-352.

6. Ponce C. Current situation of Chagas disease in Central America. Mem Inst Oswaldo Cruz 2007; 102(supl1):41-44.

7. Guhl F, Aguilera G, Pinto N, Vergara D. Actualización de la distribución geográfica y ecoepidemiología de la fauna de triatominos (Reduviidae:Triatominae) en Colombia. Biomédica 2007; 27(1):143-162.

8. Reyes M, Angulo VM, Sandoval CM. Efecto tóxico de B-cipermetrina, deltametrina y fenitrotión en cepas de Triatoma dimidiata (Latreille, 1811) y Triatoma maculata (Ericsson, 1848) (Hemiptera, Reduviidae). Biomédica 2007; 27(supl.1):75-82.

9. Angulo VM. Comportamiento de Triatoma dimidiata: un reto para su control. En: Resúmenes XII Congreso de Parasitología y Medicina Tropical. Bogotá. Biomédica;2005a:80-82.

10. Angulo VM. Ensayo de estrategias y vigilancia de Triatoma dimidiata, en Colombia. En: Guhl F (Ed.). Memorias Primer Taller internacional sobre control de la Enfermedad de Chagas. Bogotá. Universidad de los Andes. 2005b:91-102.

11. Angulo VM, Reyes M. Ciclo de vida de Triatoma dimidiata Latreille, 1811 (Hemiptera:Reduviidae) en condiciones de laboratorio: producción de ninfas para ensayos biológicos. Biomédica 2009; 29(1)119-126.

12. Ramírez CJ, Jaramillo CA, Delgado MP, Pinto NA, Aguilera G, Guhl F. Genetic structure of sylvatic, peridomestic and domestic populations of Triatoma dimidiata (Hemiptera:Reduviidae) from an endemic zone of Boyacá, Colombia. Acta Tropica 2005; 93:23-29.

13. Christensen HA, Sousa OE, Vasquez AM. Host feeding profiles of Triatoma dimidiata in peridomestic habitats of western Panamá. Am J Trop Med Hyg 1988; 38(3):477-479. 
14. Quintal RE, Polanco G. Feeding preferences of Triatoma dimidiata maculipennis in Yucatan, Mexico. Am J Trop Med Hyg 1977; 26(1):176-178.

15. Zeledón R, Calvo N, Montenegro VM, Lorosa ES, Arévalo C. A survey on Triatoma dimidiata in an urban area of the province of Heredia, Costa Rica. Mem Inst Oswaldo Cruz. Rio de Janeiro 2005; 100(6):607-612.

16. Calderón-Arguedas $\mathrm{O}$, Chinchilla M, García F, Vargas M. Preferencias alimentarias de Triatoma dimidiata (Hemiptera: Reduviidae) procedente de la meseta central de Costa Rica a finales del siglo XX. Parasitología al Día 2001; 25(3-4):78-81.

17. Zeledón R. Biología, ecología y etiología: Alimentación y preferencias alimentarias. En: EI Triatoma dimidiata y su relación con la enfermedad de Chagas. San José, Costa Rica, Editorial Universidad Estatal a Distancia;1981. p.79-87.

18. Farfán $A E$, Gutiérrez R, Sandoval CM, Castellanos J, Rodríguez L, Angulo VM. Estandarización de la prueba inmunoenzimática ELISA para la identificación de las fuentes alimentarias de los triatominos en Colombia. En: XII Congreso Colombiano de Parasitología y Medicina Tropical; Nov. 3-6; Bogotá: Biomédica. 2005;25(supl 1):93-94.

19. Farfán $A E$, Gutiérrez R, Angulo VM. ELISA para la identificación de los patrones alimentarios de Triatominae en Colombia. Rev Salud Pública, Bogotá 2007; 9(4):602-608.

20. Angulo VM, Esteban L. Nueva trampa para la captura de triatominos en hábitats silvestres y peri-domésticos. Biomédica. 2011; 31(2). Publicación anticipada en línea. http:// 190.27.195.165:8080/?idcategoria $=84719$

21. Lent H, Wygodzinsky P. Revision of the Triatominae (Hemiptera, Reduviidae) and their significance as vector of Chagas disease. Bull Am Mus Nat Hist 1979; 163:127-250.

22. Microsoft ${ }^{\circledR}$ Office Excel 2003. Parte de Microsoft Office Professional Edition; 2003.

23. Stata corp. Stata Statistical Software: Release 9,1. Collage Station, TX: StataCorp LP; 2005.

24. Weitz B. Identification of blood meals of blood-sucking arthropods. Bull World Health Organ 1956; 15:473-90. 